\title{
TEACHING POWER ELECTRONICS FROM THE CONTROL POINT OF VIEW
}

\author{
José Carlos Rodrigues de Oliveira*, Giri Venkataramanan ${ }^{* *}$ \\ *Depto. de Engenharia Eletrônica - Escola de Engenharia da UFMG \\ Belo Horizonte, MG, Brasil \\ jcarlosr@ufmg.br \\ *** Depart. of Electrical and Computer Engineering, University of Wisconsin - Madison \\ Madison, WI, USA \\ giri@engr.wisc.edu
}

\begin{abstract}
This paper presents a methodology to teach Power Electronics from the Control point of view, to be used in a laboratory course in the Undergraduate Program of Control and Automation Engineering at Federal University of Minas Gerais, Brazil. In this case, the ideal representation of the power switch is applied, not being necessary to take into consideration the switching ripple, but the averaged signals. In laboratory classes, a Microchip development module is used, permitting to study four non-isolated chopper configurations. The program covers static experimental observation and measuring, chopper modeling by equivalent averaged circuit, open-loop static calculation and validation, linearization about an operating point and dynamic non-linear and linear simulations. Due to uncertainties in the model, a suitable analog PI controller is designed and implemented from a black-box chopper model, giving a good performance to the converter under closed-loop operation. The "Motivation-by-Challenge" methodology is applied in the Power Electronics laboratory, permitting an effective participation of the students in the learning process: the closed-loop control problem of a chopper is proposed as a challenge, only finishing when a good solution is found.
\end{abstract}

Keywords - Control of Power Electronics Converters, Methodology to Teach Power Electronics, Power Electronics Teaching Laboratory.

\section{INTRODUCTION}

This paper presents a way of teaching Power Electronics from the Control point of view, employing the Motivationby-Challenge or Active Learning methodology [1, 2]. Generally, books focus their approach on Electrical Engineering students and professionals, emphasizing circuit and component design. For control purposes, it is sufficient to consider the ideal representation of the power switch, because converter main dynamics is influenced by the slowest mode established by inductors and capacitors exchanging energy, some of it dissipated as losses inside the converter. This is the reason why one must begin by a theoretical review of an R-L-C conventional circuit, before attacking the power electronic converters as well. From this approach, it is not necessary to take into consideration the switching ripple, but only the averaged signals.

Manuscript received on February 18, 2008. Revised on March 11, 2008 Recommended by the Editors of the Special Section W. Suemitsu and J. A. Pomilio.
The ideas herein presented are the result of a cooperation effort between Federal University of Minas Gerais, Brazil and the University of Wisconsin, Madison, USA [3]. The authors felt that Power Electronics instruction at UFMG could also be comprehensively taught from a control point of view, and decided to develop a new methodology which could be used in the Undergraduate Program of Control and Automation Engineering. For years, the laboratory classes in Power Electronics had been taught in the same manner as for electrical engineering students, putting strong emphasis on circuits, their principles of operation and components design, as opposed to a dynamic control point of view.

For simplification of pedagogy, as one does not need to study all types of converters in a one semester course, the choice was made upon non-isolated choppers, because their principle of operation is very intuitive, they present strong non-linearities (except for the buck converter at continuous conduction), either on continuous and discontinuous-current modes. Besides that, they can be switched at high frequencies, thus reducing size and cost of passive reactive elements, establishing a clear separation between the switching mode and the converter main dynamics.

The methodology first presents and analyzes the practical world and then uses a model (the idealized world), trying to refine it to explain reality the best it can. A typical bottleneck in this approach is that the theoretical responses (static and dynamical) often do not match to the experimental ones, although the static curves present the same qualitative behavior. The theoretical dynamics is underdamped, in opposition to the overdamped experimental one. Faced with this scenario, a suitable PI controller is designed and implemented from a black-box model, giving a good performance to the chopper under closed-loop operation. This approach is particularly valuable from the pedagogic point of view, in bringing out a practical solution to the problem at hand, in spite of model uncertainties.

As an environment for employing the methodology in the laboratory, the choice was made over a Microship low power / low cost PICDEM development module, whose flexibility is achieved by microprocessing and serial communication with a personal computer, and by the possibility of external analog circuitry adaptation, made by the user to accomplish more functions to the module.

Methodologically speaking, there must not be schizophrenia (separation) between theory, simulation and experimental circuit observation, each of them collaborating, at the right time, to put more light on the transient analysis and design of controllers for power converters. For this reason, classes in the lab follow and alternate among initial static experimental observation and measuring, chopper 
modeling by equivalent averaged circuit, open-loop static calculation and model validation, linearization about an operating point, dynamic simulation, closed-loop controller design and, finally, closed-loop experimental chopper operation and conclusions.

\section{THE MICROCHIP PICDEM MODULE}

It is constituted by the development system PICDEM MSC1 [4], whose Mother Board is based on the Microship PIC16C782 8-bit microcontroller, including a RS-232 interface and a 9 VCC power source. A firmware is embedded, with a monitor to control the CPU and the communication with a PC. A software, part of the development system, and a proper interface permit the user to access the CPU and its peripherals, for real-time configuration and for establishing operational parameters and set-point changing. The PIC microcontroller has analog and logical ports, a pair of 8-bit A/D and D/A converters, two logical output comparators, one operational amplifier, two timers and a device for generating the PWM signal.

The mother board is connected to a SMPS Daughter Board [5] through a 40-pin connector, as shown in Figure 1. The boards and the $9 \mathrm{~V}$ power source were put together in a wood platform, with sufficient space for the addition of external circuitry, like another complete +/- 5VDC regulated source, as well as connectors and potentiometers for the controller parameters adjustment. The daughter board permits to implement four different choppers, buck, boost, inverted and non-inverted buck-boost, depending on appropriate jumper connections. Figure 2 presents the daughter board circuit, with two complimentary $\mathrm{P}$ and $\mathrm{N}$ channel MOSFETs, two gate drivers, one inductor, two switching diodes, two output filtering capacitors and a lamp as the chopper load.

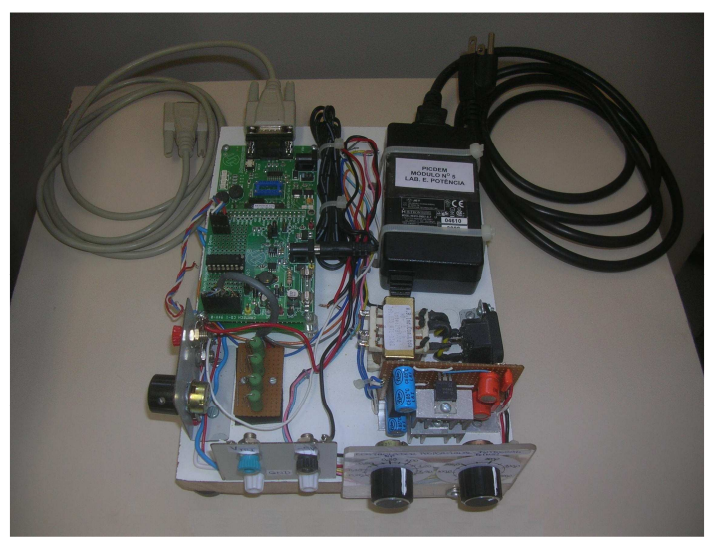

Fig. 1. Complete power converter kit.

A 5 VCC regulator feeds the PIC16C782 microcontroller through the connector. This board has an expansion area, for customized circuits to be added. To enhance the "power" board capability, four independent $150 \Omega$ resistors were added to the platform, which may be jumped one by one in parallel with the lamp, permitting to have five different values for the load.

The daughter board of Figure 2 has a current protection, implemented with the $\mathrm{R}_{11}$ resistor and a first order filter, whose output is fed back to the mother board through the 40pin connector. There are also two possibilities of output voltage feedback (inverted or non-inverted) by means of voltage dividers $R_{10}-R_{14}$ or $R_{9}-R_{13}$.

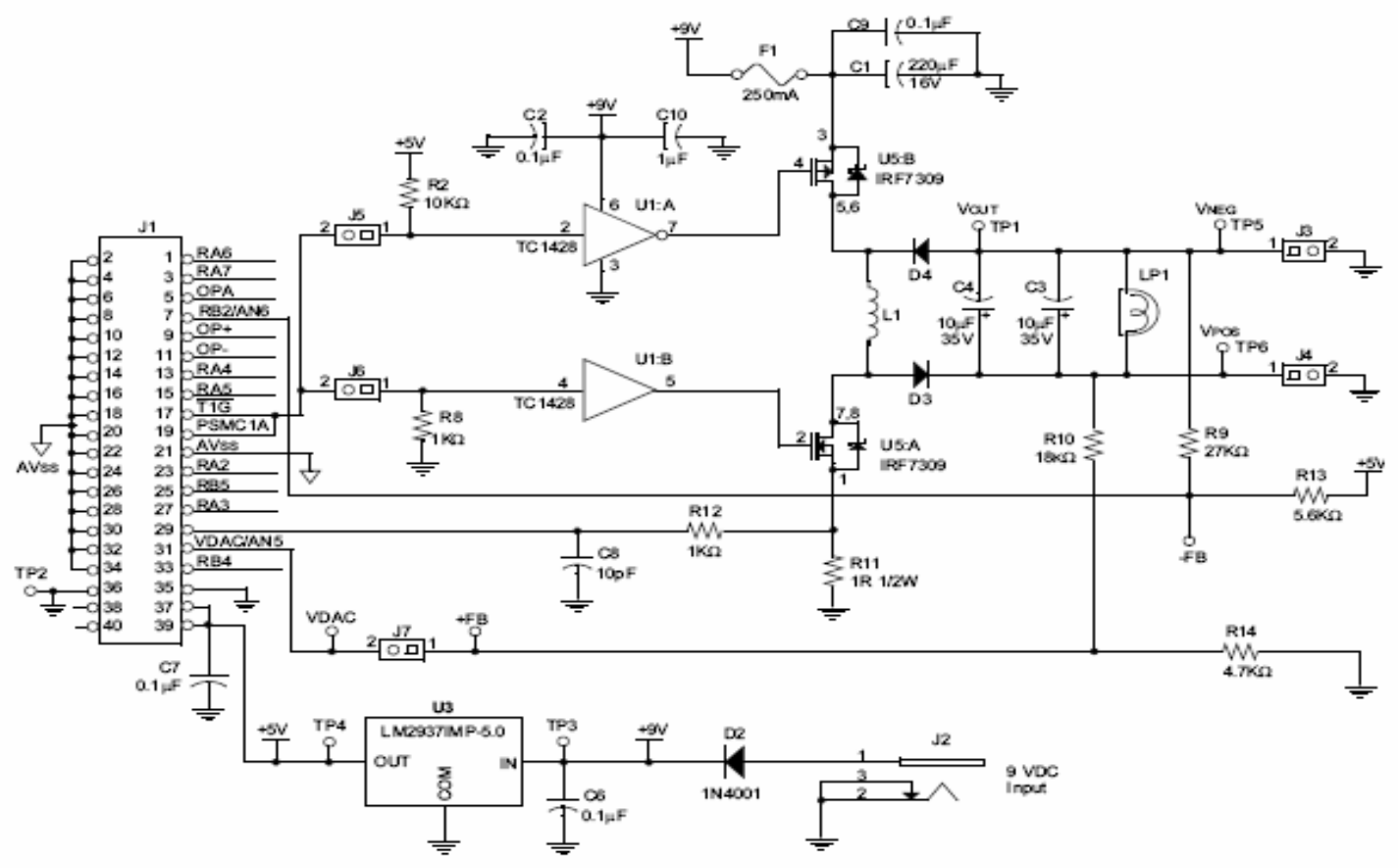

Fig. 2. Microchip SMPS Daughter Board circuit. 


\section{CHOPPER PWM CONTROL}

Figure 3 shows the circuit for generating the PWM output control signal, by logical comparison of an external analog triangular $\mathrm{V}_{\mathrm{TRI}}$ signal to the control voltage $\mathrm{V}_{\mathrm{DAC}}$, furnished by the PIC internal D/A converter. This control signal comes from the PICDEM interface on the PC, and is established by the user, when in open-loop, or by a controller, when in closed-loop. Both $\mathrm{V}_{\mathrm{TRI}}$ and $\mathrm{V}_{\mathrm{DAC}}$ must vary between 0 and $+5 \mathrm{~V}$, to adjust the chopper duty-cycle between 0 and 1 . The switching frequency $\mathrm{f}_{\mathbf{S}}$ is established externally, as well as the amplitude of $\mathrm{V}_{\mathrm{TRI}}$.

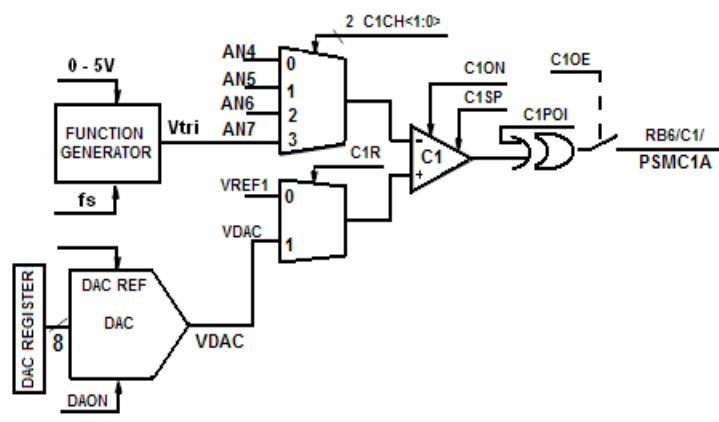

Fig. 3. Generation of the PWM signal.

The PICDEM software must be running on the PC, with its "DAC" window opened to establish the control voltage $\mathrm{V}_{\text {DAC }}$. To operate in open-loop it is necessary to load OpenLoopTri.val file into the microcontroller, from the PC, to configure the analog ports, the comparator and the other devices shown in Figure 3. The chopper begins to run after an order, given by the user to the PC (Options - Enable Eval(uation) Board).

\section{THE METHODOLOGY}

The sequence of experimental classes that have been taught for four semesters at UFMG is:

1) Introduction to Power Electronics and presentation of the methodology.

2) Familiarization with PICDEM and experimental determination of the chopper static characteristic.

3) R-L-C circuit analysis and Chopper dynamic modeling by averaging. Steady-state calculations and plotting of the static characteristic. Model validation.

4) Chopper linearization around an operation point. Transfer Function.

5) Simulation of chopper dynamic response, with the nonlinear and the linearized model.

6) Practical determination of the chopper dynamical response. Black-box dynamical modeling and comparison to the linearized model.

7) PI Controller design and chopper closed-loop simulation.

8) PI Controller implementation and observation of the closed-loop chopper dynamic response.

For each chopper configuration one must chose a set of proper connections in Figure 2. For instance, in a boost converter it is necessary to open jumpers $\mathrm{J} 4, \mathrm{~J} 5$ and $\mathrm{J} 7$ and to close J3 and J6, so that the upper MOSFET will be conducting all the time and the lower one will be switched through jumper J6 and the gate driver. In this configuration, pin TP6 will be the positive output of the converter, with respect to ground.

For modeling the chopper, the key is to begin the study with a linear R-L-C circuit, as converters have inductors and capacitors for temporary energy store, as well as losses which may be represented by resistors. After that, the chopper space-state model is settled in a third-order structure, to take into account a filter capacitor $C_{b}$ put in parallel with the input DC source, generally constituted by a non-controlled rectifier. An equivalent averaged circuit is used, as presented in Figure 4 for the boost converter: the static switch and diode are replaced by voltage and current sources, function of the duty-cycle D and, respectively, of the output voltage $\mathrm{V}_{\mathrm{O}}\left(\right.$ or $\left.\mathrm{V}_{\mathrm{CO}}\right)$ and of the inductor current $\mathrm{I}_{\mathrm{L}}$.

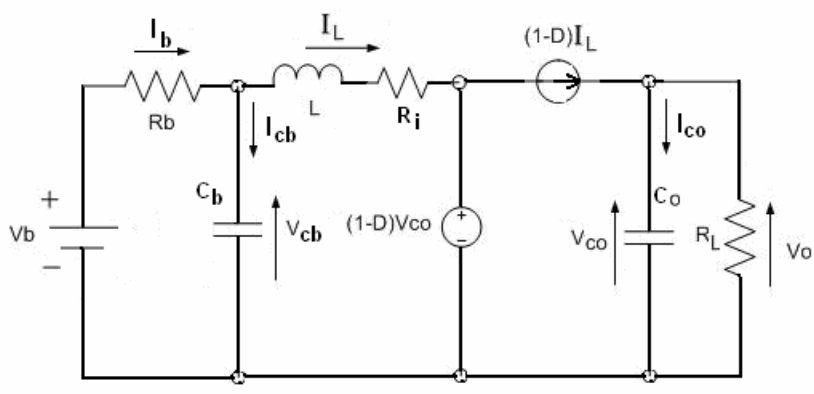

Fig. 4. Boost equivalent averaged circuit.

To better model the real chopper, an output resistance $R_{b}$ is added to the DC source $V_{b}$, as well as a series resistance $R_{i}$ in the inductor.

In Figure 4, all variables are considered in their averaged (DC) values, so they are presented in capital letters. This approach, neglecting the ripple in voltages and currents, is sufficient for control purposes.

Applying the Kirchhoff current law to the nodes on top of the two capacitors, and the Kirchhoff voltage law around the loop constituted by capacitor $\mathrm{C}_{\mathrm{b}}$, the inductor and the voltage source, a state-space equation may be written:

$$
\begin{aligned}
\frac{d}{d t} X & =A X+b U \\
y & =c . X
\end{aligned}
$$

where:

$$
\begin{gathered}
X=\left[\begin{array}{lll}
I_{L} & V_{C b} V_{C O}
\end{array}\right]^{T} \\
U=V_{b} \quad \text { and } \quad y=V_{O}=V_{C O}
\end{gathered}
$$

From the control point-of-view, the chopper may be represented by the block diagram of Figure 5 below.

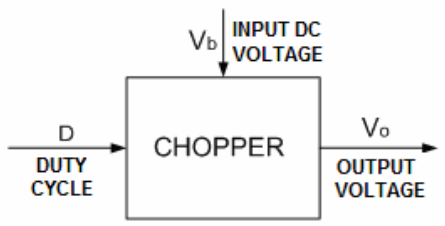

Fig. 5. Chopper block control diagram. 
In the diagram of Figure 5, D (duty-cycle) is the control input, $V_{b}$ is a perturbation input, and $V_{O}$ is the controlled variable. The model thus obtained is non-linear, as matrix $\mathbf{A}$ is function of the input variable $\mathrm{D}$, except for the buck converter operating in continuous-current mode.

Open-loop static calculations are done by considering the left term of (1) equal to zero, $\mathrm{V}_{\mathrm{b}}$ and $\mathrm{D}$ constant, and multiplying this expression by the inverse of the system matrix:

$$
X=-A^{-1} b U
$$

For each value of $\mathrm{D}, \mathbf{A}$ is constant and the system is linear. After estimating/measuring the numerical values of all model parameters, the state vector $\mathrm{X}$ can be calculated for a variety of duty-cycles from 0 to 1 , in increments of 0.01 , using Matlab. This permits to plot the theoretical static characteristics $\mathrm{V}_{\mathrm{O}} \times \mathrm{D}, \mathrm{I}_{\mathrm{L}} \times \mathrm{D}$ and $\mathrm{V}_{\mathrm{CB}} \times \mathrm{D}$.

At this point, one must validate the chopper averaged model, by comparing the theoretical and experimental static output characteristics. Certainly, discrepancies appear, because models only approximate reality. The initial parameters used in the model must be adjusted, in a trial-anderror basis, to match the calculated curve to the real one. Figure 6 shows this validation for the PICDEM boost converter, with a good agreement in the duty-cycle range from 0.00 to 0.70 .

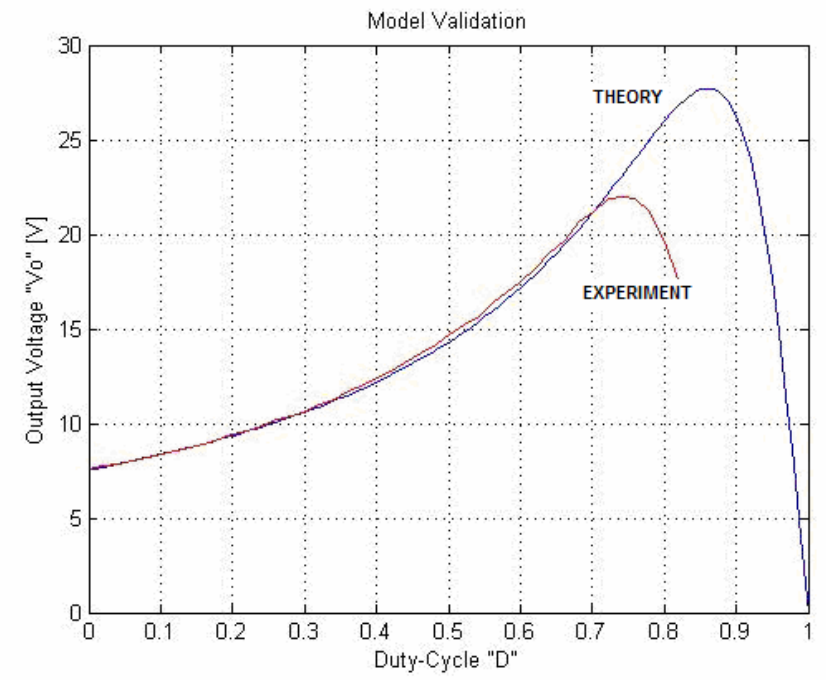

Fig. 6. Boost converter theoretical and experimental characteristics.

In the trial-and-error procedure presented above, $\mathrm{V}_{\mathrm{b}}$ was refined at $D=0, R_{b}$ and $R_{i}$ have influenced all the theoretical curve including its peak. It was noted that a greater value of load resistance $\mathrm{R}_{\mathrm{L}}$ strongly increases the calculated peak of $\mathrm{V}_{\mathrm{O}}$. As the model was calculated by (5) for the converter DC steady-state, changes in capacitances $C_{b}$ and $C_{O}$ and in the inductance $\mathrm{L}$ do not have any influence on this theoretical characteristic. For duty-cycles greater than 0.70 the inductor current increases so much and more losses not taken into account must be the reason for the lower experimental peak. In fact, the model does not compute losses on the two diodes D3 and D4 of Figure 2, as well as those on the two MOSFETs.

A challenge may be put to the students: they can be asked to calculate the input power furnished by the DC source, the losses in the source and converter, the output power transferred to the load and the overall system efficiency. Plotting these variables against duty-cycle will clearly show the better operating range for the chopper.

As far as the converter model is non-linear, it is convenient to linearized it about an operating point, defined by the input vector $\mathrm{U}_{\mathrm{O}}=\left[\mathrm{D}_{\mathrm{O}} \mathrm{V}_{\mathrm{bO}}\right]$ and by the state vector $\mathrm{X}_{\mathrm{O}}$ $=\left[\mathrm{I}_{\mathrm{Lo}} \mathrm{V}_{\text {cbo }} \mathrm{V}_{\text {coo }}\right]$. Calling $d, v_{b}, v_{o}, i_{L}$ and $x$ the small variations, respectively to the operating point DC variables, one can write:

$$
\frac{d}{d t} x=A_{O} x+b_{1} v_{b}+b_{2} d
$$

Figure 7 presents the linearized block diagram. For each pair output/input a transfer function is defined, supposing the other input equal to zero. Those four transfer functions can be obtained with Matlab, from the state-space model (6), with:

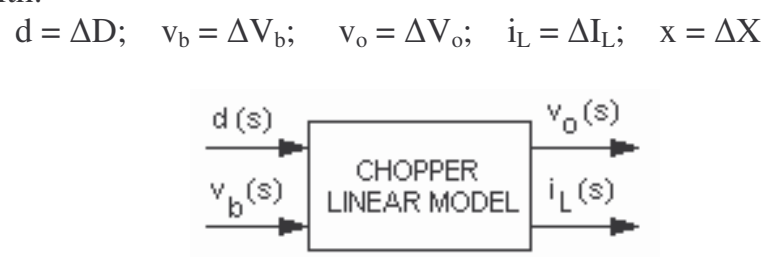

Fig. 7. Converter linearized diagram.

The students are asked to plot the poles and the zeroes of the transfer functions in the "s" plane, so they can estimate the converter dynamics face to small variations in the inputs. A pair of complex poles appear, and even zeroes (depending on the output/input considered), which preview an oscillating transient response. Dynamic simulations with both the nonlinear model (1) and the linear model (6) must be carried with Matlab.

There are discrepancies between the analytical predictions and the empirical measurements, as it is observed an experimental overdamped dynamics. This could be due to a current limitation in the source current from the power supply, that makes the inductor current not be a "real" state variable, or due to discontinuous-conduction operating mode. In fact, chopper experimental static characteristic is obtained almost from 0 to 1 duty-cycle, thus taking into consideration also discontinuous-current modes, although the theoretical averaged model has only been based in the continuouscurrent operation.

There is, again, more power dissipation in the real circuit than considered in the model and, certainly, this might explain the overdamped performance observed experimentally. Referring to the boost chopper, some computer calculations putting additional serial resistance in the output capacitor have changed the static characteristic, lowering its peak; but the very high resistance values used were not compatible to real series capacitor resistances. Additionally, the two diodes and mosfet together have a voltage drop of about 2 volts in their on-state, and this could easily change the results, since one is working with a $9 \mathrm{~V}$ power supply. Polivka et al [6] provide systematic example of modeling parasitic elements from the inductor, mosfets, 
diodes, etc. to account for discrepancies. This might be a good and not trivial challenge to be proposed to the students!

To bypass those drawbacks, a step response black-box modeling may be taken, permitting to design a suitable closed-loop controller for the chopper. Yet in open-loop operation, the students are asked to observe the dynamical response to a small step variation, applying a square wave pattern by means of an "Arbitrary Waveform", previously stored in the PC interface. It is possible to adjust the square wave repetition rate, permitting the user to match its halfperiod to the chopper response time.

The controller design approach is compatible to the analog design techniques studied in the curriculum. Choosing a Proportional plus Integral Controller (PI):

$$
G_{C}(s)=k_{P}\left(1+\frac{1}{T_{i} s}\right)=\frac{k_{P}\left(1+T_{i} s\right)}{T_{i} s}
$$

the Root-Locus Method can be easily employed for the controller direct synthesis. In the case of a chopper overdamped model, the PI zero $\left(-1 / \mathrm{T}_{\mathrm{I}}\right)$ could be chosen equal to the dominant converter pole $(-1 / \mathrm{T})$, and the controller gain $\mathrm{k}_{\mathrm{P}}$ adjusted to make the closed-loop response two times faster (at least, theoretically), as in Figure 8. A derivative action is not recommended, because of the inherent noise due to the high-frequency switching.

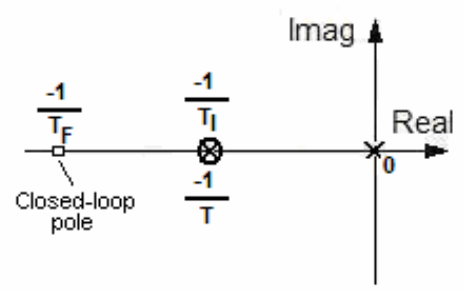

Fig. 8. PI controller design.

Regarding to the experimental module, although PICDEM has one internal Operational Amplifier, the Proportional plus Integral controller can be better implemented by an external circuit, with four Op. Amps., as shown in Figure 9.

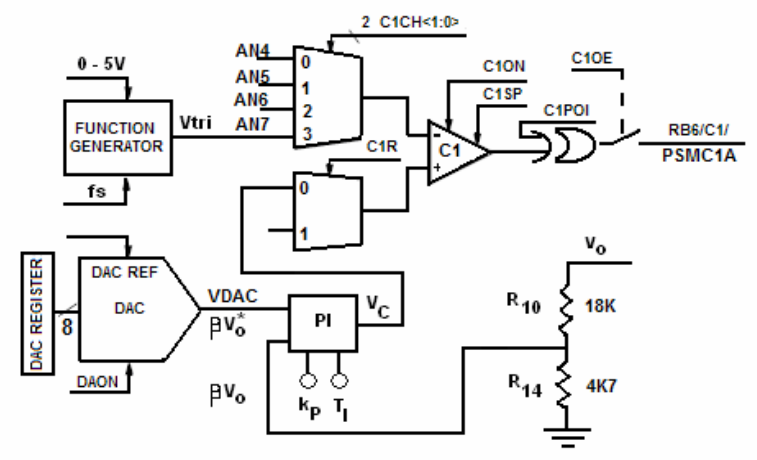

Fig. 9. Analog PI controller coupled to the PWM generation.

Now the PC interface must establish the set-point for the chopper output voltage, and both the feedback voltage and the reference one must be normalized to the $0-5 \mathrm{~V}$ range, using a factor $\beta$ to maintain compatibility with the output of the PICDEM internal DAC device. In this case, the control voltage $\mathrm{V}_{\mathrm{C}}$, to be compared to the triangular waveform, is furnished by the output of the PI controller. This approach permits the user to tune the controller proportional gain $\mathrm{k}_{\mathrm{P}}$ and the integral time-constant $\mathrm{T}_{\mathrm{I}}$ independently, by means of two potentiometers. Loading ClosedLoopTri.val file from the PC into the microcontroller reconfigures the analog ports, comparator and other devices shown in Figure 9.

After implementing the PI controller, students must verify whether the chopper output voltage $V_{O}$ tracks itself to several values of the reference $\mathrm{V}_{\mathrm{O}}{ }^{*}$, in a static observation. For the dynamical response, besides tuning the pair $\left(\mathrm{k}_{\mathrm{P}}, \mathrm{T}_{\mathrm{I}}\right)$ obtained in the controller design, the students are encouraged to try other combinations, acquiring the chopper output voltage with a digital oscilloscope, also linked to the PC through a second serial interface. In this way, the reference voltage step and the chopper output may be plotted together, permitting to match the controller design on a trial-and-error basis by observing the closed-loop dynamical performance. Figure 10 presents the response of the PICDEM boost converter for two sets of PI tuning. As the signals were got from the reference and the feedback inputs to the controller, they are scaled by the factor $\beta=$ 0.20 , meaning a change in output voltage from 10.00 to 11.00 Volt.

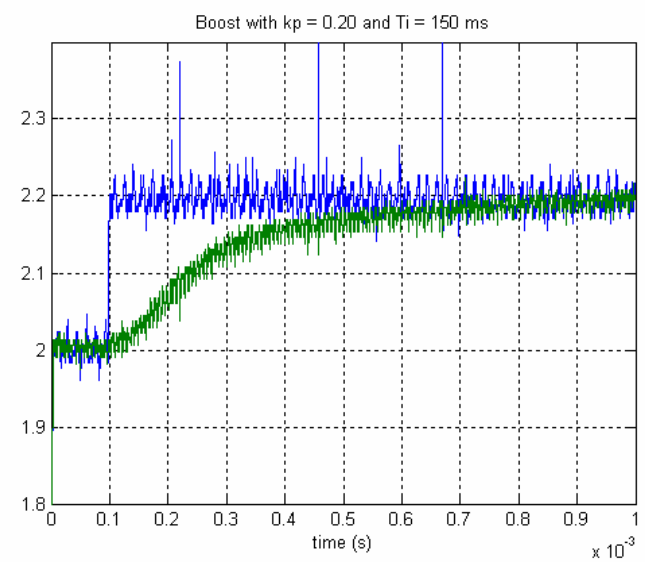

(a)

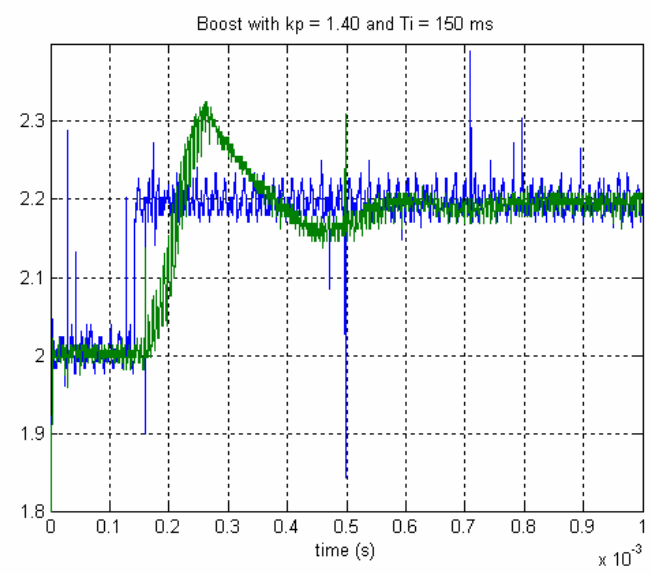

(b)

Fig. 10. Boost closed-loop responses:

(a) $\mathrm{k}_{\mathrm{P}}=0.20$ and $\mathrm{T}_{\mathrm{I}}=150 \mathrm{~ms}$

(b) $\mathrm{k}_{\mathrm{P}}=1.40$ and $\mathrm{T}_{\mathrm{I}}=150 \mathrm{~ms}$ 


\section{PLANNING THE COURSE AND EVALUATING THE STUDENTS PERFORMANCE}

In planning a lab course, one must consider its objective, the learning methodology, the course tasks and their chronology, and finally the form of evaluation, all of them coherently with the adopted methodology. To establish the objective, it is necessary to have in mind what are the abilities expected to be developed by the students. Besides improving technical skills, they need to find satisfaction and to grow in self-confidence and initiative. Although the course may be oriented to a medium profile student, it shall also introduce the liking for research and development, and the motivation for more advanced studies [7].

The difference between the proposed methodology and conventional approach is the way the lab course is conceived and developed: the "challenge" versus the "ready solutions", real world problems versus prepared examples. In the topdown (conventional) method, the entire subject is assimilated by the students in a fragmentary way, and they finish the course not knowing exactly how to join all the parts of the presented knowledge for the solution of real problems. On the opposite side, the Motivation-by-Challenge considers the program items as tools (important, but just tools) to solve real engineering problems. The chronology must be suited in accordance to the challenge, its steps and the solutions.

The evaluation of the students is the feedback of the learning effectiveness. The final goal, the real learning, will be confirmed by the quality of the solutions proposed and developed for the challenge. At the laboratory, conventional written tests may be applied, but with a smaller emphasis. The evaluation must be conducted continuously in time, profiting from the small number of students the instructor has in each session, permitting to closer follow their progress in attacking the challenge. Partial and full reports are mandatory, together with oral presentations while operating the experimental module.

\section{CONCLUSION}

The methodology applied to the Power Electronics Laboratory permits an effective participation of the students in the learning process. The control problem of a chopper is proposed as a challenge, only finishing when a good solution is found.

After four semesters adopting this new methodology in the Undergraduate Program of Control and Automation Engineering at Federal University of Minas Gerais, Brazil, more satisfactory learning results have been obtained, demonstrated by a greater involvement of students and some teachers. The students have shown more interest in the course, going beyond the conventional established goals. The great challenge presented to the teacher is to create new real control challenges in the laboratory. Trained and motivated people and financial / material resources are indeed essential.

Although statistical data have not yet been collected from the application of this methodology, preliminary results show that it is improving the quality of the learning process, when compared to the conventional approach. And the basic key for the success of this idea is to believe and to invest on it!

\section{ACKNOWLEDGEMENT}

The authors acknowledge the financial support received from the Brazilian Council CNPq for the Visiting Researcher Scholarship granted to Prof. Venkataramanan during his sabbatical at UFMG.

\section{REFERENCES}

[1] N.A. Kheir, K.J. Åström et al, "Control Systems Engineering Education”, Automatica, IFAC, vol. 32, no. 2, pp. 147-166, 1996.

[2] J. J., Zhu, "Motivation-by-Challenge for Teaching Control Systems", IEEE Control Systems, vol. 14, no. 5, pp. 64-68, October, 1994.

[3] J. C. R. Oliveira, G. Venkataramanan, "Desenvolvimento e Aplicação de Metodologias Centradas no Aprendizado Ativo para Ensino e Pesquisa em Eletrônica de Potência e Acionamentos Elétricos" (Development and Application of Active Learning Methodologies for Teaching and Research in Power Electronics and Electrical Drives), $C N P q$ Research Project no. 308863/2005-4, Escola de Engenharia da UFMG, Feb-May, 2006.

[4] www.microchip.com, Microchip Technology, Inc., "PICDEM MSC1 User's Guide", 2001.

[5] www.microchip.com, Microchip Technology, Inc., "PICDEM MSC1 SMPS Daughter Board User's Guide", 2003.

[6] W.M.Polivka, P. R. K. Chetty, R. D. Middlebrook, "State-Space Average Modeling of Converters with Parasitics and Storage-Time Modulation", Proc. IEEE Power Electron. Spec. Conf., 1980, pp. 119-145.

[7] L.G.A. Scott, "Aplicação da Metodologia "Motivação pelo Desafio" ao Ensino de Controle de Sistemas Lineares" (Application of the Methodology "Motivation-by-Challenge" in Teaching Control of Linear Systems), MS Thesis, UFMG, Brazil, December, 1998.

\section{BIOGRAPHIES}

José Carlos R. de Oliveira, born in Belo Horizonte, MG, Brazil, in 1951, is Electrical Engineer, UFMG, Brazil (1974), Master of Science in Electrical Engineering, UFMG (1980) and Doctor in Electrical Engineering, INPT, France (1991).

He was engaged in international research cooperation between UFMG and INPT, Toulouse, France, from 1979 to 1991, and hosted Prof. Venkataramanan during part of his sabbatical period in 2006. Presently, he is Associated Professor in the Department of Electronics Engineering at Federal University of Minas Gerais, Brazil, coordinator of the teaching labs in Process Control, and has worked for the International Relations Board of UFMG. His main fields of interest are the application of methodologies for teaching Power Electronics and Control of Industrial Processes, Instrumentation and Control of Electrical Drives.

Dr. Oliveira is a member of SOBRAEP - Brazilian Society on Power Electronics and SBA - Brazilian Society on Control and Automation. During the period 1982-1984 he 
was the Regional Director of SBA for Minas Gerais and Espírito Santo, as well as member of its national technical and administrative board. He has participated on the organization of SOBRAEP's Brazilian Conference on Power Electronics - COBEP'97 in Belo Horizonte, MG, Brazil, and actuated as a reviewer for SOBRAEP and SBA conferences.

Giri Venkataramanan, born in India in 1964, is BE on Electrical and Electronics Engineering, University of Madras, India (1985), Master of Science in Electrical Engineering, California Institute of Technology (1987), and $\mathrm{PhD}$ in Electrical Engineering, University of Wisconsin Madison (1992).

Presently, he is Associated Professor in the Department of Electrical and Computer Engineering, University of Wisconsin-Madison, USA, as well as Associate Director of WEMPEC - Wisconsin Electric Machines and Power Electronics Consortium. He was Assistant Professor (19921998) and Associate Professor (1998-1999) at Montana State University - Bozeman. His main fields of interest are power electronics, electromagnetic compatibility, electronics packaging, power engineering and education.

Dr. Venkataramanan is a member of IEEE, where he was Secretary/Treasurer of the Montana Section (1996-1997) and Vice-Chair (1998-1999). He joins IEEE Power Electronics Society, Electromagnetic Compatibility Society, Electronics Packaging Society, Power Engineering Society and Education Society. He has been engaged in program organization and technical program committee of many IEEE Conferences. He is the recipient of the 2002 Gerald Morgridge Teaching Award, Department of ECE of UW, has five US Patents and has taken consultant activities for many companies in the US. 